\title{
What Will The Post-Pandemic City Look Like?
}

\author{
Michael Batty ${ }^{1} \odot \otimes$ \\ ${ }^{1}$ Centre for Advanced Spatial Analysis (CASA), University College London (UCL) \\ Keywords: hypothetical-city, lockdown, compactness, decentralisation, gravitation, london, centralisation \\ https://doi.org/10.32866/001c.23581
}

Findings

It is impossible to predict the future spatial structure of cities from our current models of location and travel behaviour for the forces that have previously determined where people locate, have been turned on their heads by the pandemic. The only way we can explore such an uncertain future is to build hypothetical cities on which we are able to experiment with different urban futures. Here we build a hypothetical, symmetric, monocentric city which we proceed to lockdown by moving 80 percent of its workers to work from home. We then illustrate how we can relax this situation, letting the city return to a new equilibrium and showing how changes in our travel behaviour can lead to very different forms of future city. We argue that only through hypothetical simulations can we explore this future effectively and we point to where we have applied the model to London.

\section{Questions}

The big question is contained in the title. The recent pandemic has changed patterns of location and travel in the city so fundamentally that our current theories and models are less able than ever in providing us with informed predictions of what the future spatial structure of our cities will be like. The pandemic and the deep lockdown that many cities have faced during the last year will surely end at some point soon we hope. We will control the disease through vaccines and various other regulations on our behaviour that will probably lead to some sort of herd immunity and the simplest prediction is that we will return to the 'old normal'. People will no longer work at home and the current increase in demand for low density living will revert to the slow compaction of large cities that was evident prior to the pandemic.

Many commentators think, however, that a 'new normal' will emerge and that the penchant for doing many things from home - working, shopping, and learning using internet technologies - will not disappear, but is here to stay in one form or another and will have significant impacts on urban location. In short, many argue that we have no idea of what the future will hold in terms of where we will locate and how we will travel in cities. Usually when we make predictions, we base our logic on what has happened in the past but the current logic is no guide to the future as it represents a deep unprecedented reaction posed by the fear of infection in high density environments. In this sense, it is unlike past plagues, and every plague has been different with respect to how we respond and restore our past behaviours once the pandemic passes. Of course, it is arguable that even before the pandemic, our predictions using the modelling technologies that have developed over the last fifty years were deeply flawed (Batty 2018). 
So what is the answer to the question? I am inclined to say your guess is as good as mine but there is some hope. We do have a sense in which the forces that make us locate centrally in higher density locations or in lower density suburban locations are understood. Nearly 60 years ago, one of the great intellectual leaders of planning, Catherine Bauer Wurster (1963) wrote an essay entitled 'The Form and Structure of the Future Urban Complex' in which she argued that the form of future cities would reflect the age old tension between the forces of centralisation and decentralisation. She argued these forces are never simple and there are contradictory trends in both directions. Cities are built up in historical layers that continue to compact and there is a sense in which the path dependence that this history implies is such that centralisation and decentralisation will continue as two forces in complex balance.

\section{Methods}

How then do we proceed to an informed discussion of this future using the tools that we have for predicting changes in locational and travel behaviour in large cities? The methods we propose are based on constructing a hypothetical city which leads to a 'toy' model built on plausible assumptions about the various forces that we are able to explore as different scenarios work themselves out. To explore how people might move from this lockdown to a new normal, we first lock down our hypothetical city and its model, assuming a large proportion of workers work from home; then we gradually release the lockdown, slowly perturbing it to generate new patterns that might pertain to this future. In Fig 1a, we construct this city on a square symmetric $11 \times 11$ grid and we generate a distribution of where people live and work in Fig $1 \mathrm{~b}$ using the simplest gravitational model based on the inverse square law. This reinforces the symmetry around the centre of the grid where the distribution of where people live and work is identical. We then change the distribution of work by imposing a polycentric hierarchy of high density hubs on this landscape shown in Fig $1 \mathrm{c}$ and we derive a plausible trip distribution using a doubly constrained gravity model which produces a consistently structured hypothetical city. We then lock it down by assuming that $80 \%$ of workers work at home and we end up with work redistributed as we show in Fig 1d.

We make the assumption that this locked down picture begins to change as people re-evaluate the location of their homes and work according to the new gravitational field that results, thus producing a new landscape. We start with the locked down home and work distributions in Fig $1 \mathrm{~b}$ and $1 \mathrm{~d}$, throw in a substantial amount of noise to perturb the symmetry of the landscape, and then run the new model. This produces the landscapes in Figs 1e and 1f for home and work and we continue this process of distribution iteratively until a long term equilibrium emerges. This centralises the landscape of work and home quite dramatically with the city beginning to compact, almost collapsing in on itself which we show in the 'implosions' in Figs $1 \mathrm{~g}$ and $1 \mathrm{~h}$. 


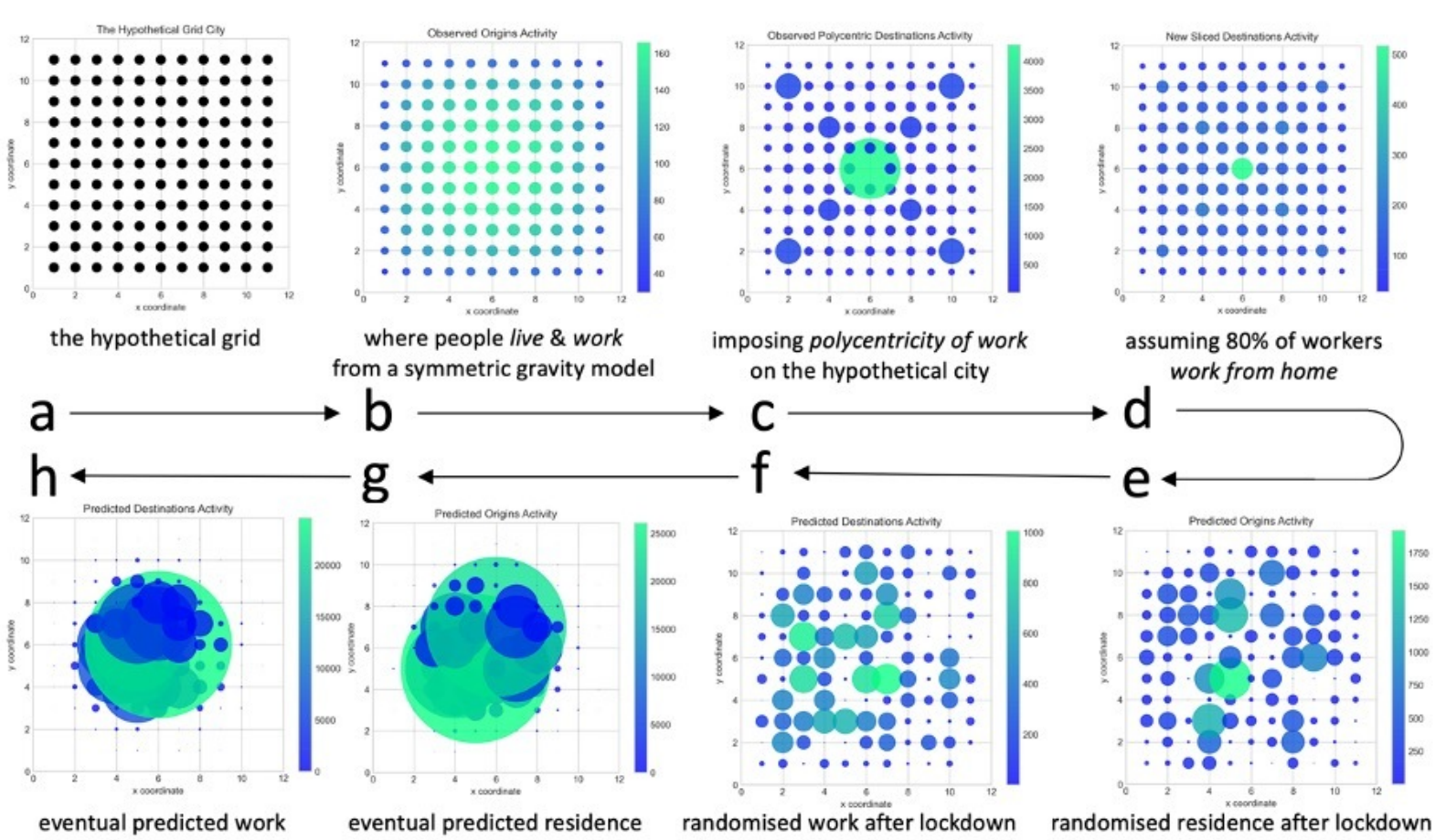

Figure 1. The Hypothetical Grid City in Lockdown and Readjustment as Lockdown is Lifted

You might say none of this is surprising in that the city we have constructed is completely symmetric, monocentric around its centre, and the gravitational model with its inverse deterrence functions to travel reinforces this. Nevertheless it is surprising that the effects of symmetry and centralisation are so strong. In fact this assumes our behaviour patterns are based on the old normal. To move away from this - to accept that we might wish to be further away from everybody than in the past assuming that social distancing is scaled up across the whole city, we adopt a generic distribution based on the gamma function of distance attraction-deterrence. This enables us to generate patterns of behaviour over a very wide range of types. Some of these are shown in Fig $\underline{2 a}$ and we have explored many variants of this function under a diverse range of parameter values, enabling us to predict a wide variety of centralised and decentralised patterns. Starting from the lockdown, we are able to generate patterns as diverse as the centralised ones shown in Figs $2 \mathrm{~b}$ and $2 \mathrm{c}$ and the decentralised ones shown in Figs $2 \mathrm{~d}$ and $2 \mathrm{e}$.

\section{Findings}

Our generic finding is that with the constructed artificial city, its internal symmetry is so strong that it takes very radical change in travel patterns to really turn the city inside out. By radical change, we mean that workers have a preference for working at further and further distances from their work up to a limit when the traditional deterrent effects of distance kick back in. We have explored various scenarios that can generate such decentralisation but it takes a major change in travel behaviour, one that we consider unrealistic, for the city to explode in any fundamental way. We have computed various measures 

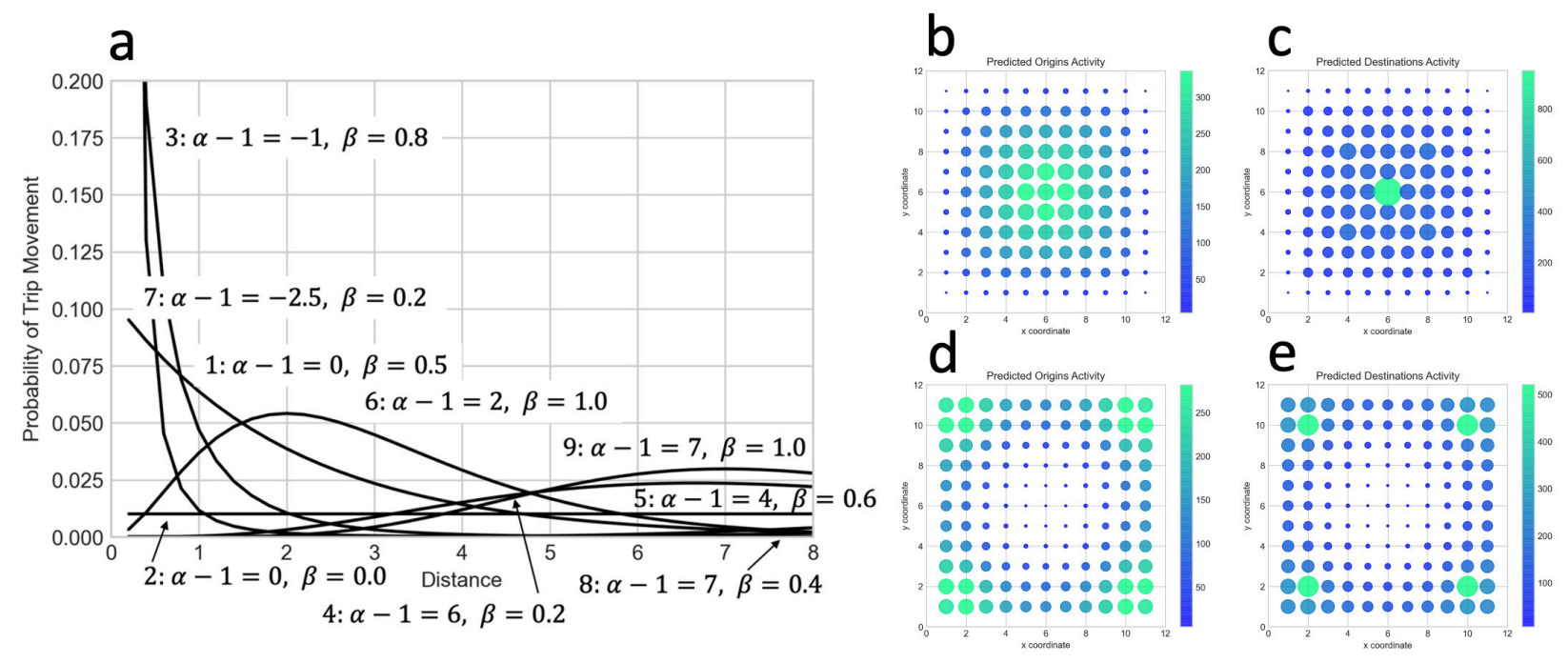

Figure 2. Changing Travel Behaviour Generating Increased Centrality to Decentrality

of these kinds of spatial shift and these are contained in the extensive material which supports this note (Batty 2020b). What we cannot easily do in our hypothetical city is break symmetry. We can introduce noise but the symmetric grid forces many potential changes in the locations of where we live and work.

In terms of the current pandemic, the big question is 'will we revert to highly centralised urban living or is this the beginning of a sea change that forces us to spread out further?' What we have shown here is the power of speculating about the future through simulation, through what we call 'computable thought experiments'. We know our simulations are not likely to be correct but at least we have a framework for exploring a range of possibilities. In reflecting on what we have done, we consider that it is unlikely that cities will explode to their edges after the pandemic ends for it will still be impossible to ignore the layers and layers of history which have built the central city historically. There may be some increase in decentralised living and there is certain to be a new mobility with respect to living and working remotely. But to make informed speculations in this way, we need to move to much bigger systems, bigger grids, which give greater variety, where we might even be able to incorporate different kinds of force and flow. We have already transferred our model to a real city, to the London metropolitan region, which enriches the analysis through its heterogeneity and relative asymmetry (Batty 2020a). But in all our experiments so far, even in a real system like London, the continued focus on the centre is difficult to break and once again our findings are that centralisation in big cities is likely to restore itself. This is despite the current debate amongst big companies that are uncertain about how far they will continue to work from central city locations. As the pandemic continues, this picture, far from getting clearer, is increasingly blurred and this reinforces our major finding that with 
what appeared in the past to be relatively simple forces at work in cities, the future betrays a level of complexity that continues to confound our ability to predict.

Submitted: March 10, 2021 AEST, Accepted: May 03, 2021 AEST

This is an open-access article distributed under the terms of the Creative Commons Attribution 4.0 International License (CCBY-SA-4.0). View this license's legal deed at https://creativecommons.org/ licenses/by-sa/4.0 and legal code at https://creativecommons.org/licenses/by-sa/4.0/legalcode for more information. 


\section{REFERENCES}

Batty, Michael. 2018. Inventing Future Cities. Cambridge, MA: The MIT Press. https://doi.org/ 10.7551/mitpress/11923.001.0001.

- - - 2020a. "The Morphology of a Post- Pandemic City: Applications to London." Working Paper 226. London: Centre for Advanced Spatial Analysis, University College London.

-__. 2020b. "The Socially-Distanced City: Speculation Through Simulation." Working Paper 225. London: Centre for Advanced Spatial Analysis, University College London.

Wurster, C.B. 1963. "The Form and Structure of the Future Urban Complex.” In Cities and Space: The Future Use of Urban Land, edited by L. Wingo Jr., 73-102. Baltimore, MD: Johns Hopkins Press. 